\title{
Weak lensing effect on CMB in the presence of a dipole anisotropy
}

\author{
Abhineet Agarwal ${ }^{1, a}$, Naveen K. Singh ${ }^{2, b}$, Pankaj Jain ${ }^{3, c}$, Prabhakar Tiwari ${ }^{4, d}$ \\ ${ }^{1}$ Centre for Theoretical Physics, Jamia Millia Islamia, New Delhi 110025, India \\ ${ }^{2}$ School of Physics and Astronomy, Sun Yat-Sen University Zhuhai, 2 Daxue Rd, Tangjia, Zhuhai, China \\ ${ }^{3}$ Department of Physics, Indian Institute of Technology, Kanpur 208016, India \\ ${ }^{4}$ National Astronomical Observatories, CAS, Beijing 100012, China
}

Received: 30 January 2019 / Accepted: 28 June 2019 / Published online: 11 July 2019

(C) The Author(s) 2019

\begin{abstract}
We investigate weak lensing effect on cosmic microwave background (CMB) in the presence of a superhorizon mode which leads to a dipole anisotropy in the CMB power spectrum and in large scale structures. The approach of flat-sky approximation is considered. We determine the functions $\sigma_{0}^{2}$ and $\sigma_{2}^{2}$ that appear in expressions of the lensed CMB power spectrum in the presence of the dipole anisotropy. We determine the correction to B-mode power spectrum which is found to be appreciable at low multipoles $(l)$, around $15 \%$ at $l=10$. However, the temperature and E-mode power spectrum are not altered significantly.
\end{abstract}

\section{Introduction}

In unveiling the dynamics and contents of the universe, cosmic microwave background radiation has been one of the essential tools. CMB constrains cosmological parameters, and it provides a path in developing modern cosmology. The precision level of the experiments for the CMB spectrum and hence the accuracy of prediction of a theoretical model has been increasing year by year. The CMB anisotropy can be determined by the Physics of last scattering surface (LSS) and the medium effects due to the propagation of photons from LSS to the observer. The primary contribution to the anisotropy is due to the strength of the metric perturbations at LSS. Some other effects such as ISW effect, reionization give additional modifications. The lensing effect is associated with the geodesics of photons from LSS to us. It can be considered as a secondary contribution in modifying CMB anisotropy, and it must be taken into account to reliably predict the CMB signal. The lensing gives a very small contri-

\footnotetext{
a e-mail: abuwdu123@gmail.com

be-mail: naveen.nkumars@gmail.com

c e-mail: pkjain@iitk.ac.in

de-mail: ptiwari@nao.cas.cn
}

bution on the reionization and the ISW signal as these are associated with the large scale. However, on degree-scale of primary acoustic peaks, weak lensing effect is expected at the order of arc minute on the CMB spectrum [1].

In this paper, we are interested in the weak lensing effect on the CMB spectrum arising due to a superhorizon mode in the Newtonian potential $[2,3]$. The unexpected observation of anisotropy at large scale leads us to study the implications of such a model. Some of the anomalies were discovered in the WMAP data [4]. These include the alignment between low multipoles [5-7], large cold spot in the southern hemisphere [8-10], hemisphere power asymmetry [11] etc. The Planck data has further confirmed the existence of such anomalies [12]. According to Planck, the dipole modulation of power lies in the range $l=2-600$ at $1.1-3.5 \sigma$. In addition, it confirmed octopole-quadrupole alignment at $98 \%$ level and a power deficit below $l=40$. Other observables, such as, radio polarization from radio galaxies and optical polarization of quasars also indicate anisotropy on large distance scales. Remarkably, orientations of dipole axis of radio polarization [13], dipole, quadrupole and octopole axes of the CMB [14] and the direction of alignment of the pattern of two-point correlation in the optical polarization $[15,16]$ all point towards the Virgo direction. In this paper we study the gravitational lensing in the model proposed by Gordon et al. [2]. The model was originally proposed in order to explain the power deficit at low $l$ and the alignment of quadrupole and octopole axes. It has also been studied in Refs. [3,17,18]. The CMB spectrum in this model has been investigated in Refs. [19-21]. We assume a long wavelength mode of the form $[3,18]$

$\psi^{d}(\vec{x})=\beta \sin (\vec{k} \cdot \vec{x}+\omega)$

with $\omega=0$. The model has 4 parameters, $\beta$ and the three components of the fixed vector $\vec{k}$. We choose the coordinate system such that the direction of this vector is $\hat{x}$ and denote its 
magnitude as $\kappa_{s}$. In Refs. $[3,18]$ it was chosen to be $\hat{z}$. In Ref. [3], it has been shown that the hemispherical power asymmetry in CMB can be obtained by a super-horizon mode generated during inflation. It is also explained how the variation of background value of scalar field across the observable Universe assists a large amplitude super horizon fluctuation in generating the power asymmetry. It further showed that a single scalar field cannot provide the observed power asymmetry without violating constraints arising due to homogeneity of the Universe. The task of generating the desired power asymmetry without violating homogeneity constraints requires additional fields, such as the curvaton field. The measured rms values of quadrupole and octupole lead to an observational constraint, $\kappa_{s}^{3} \beta \lesssim 1.26 \times 10^{-5} H_{0}^{3}$, [3,18]. The superhorizon mode also leads to a dipole anisotropy in the matter distribution at large distance scales [18].

In this paper, we study the weak lensing effect on the CMB spectrum with the flat-sky approximation in the model given in Eq. (1). The contribution of the standard weak lensing effect to the CMB power spectrum, without including the inhomogeneous term given in Eq. (1), has been computed in Refs. [22-24]. In Sect. 2, we generalize the two-point correlation function due to the weak lensing by including the super horizon mode. In this section, we also calculate the expectation value of a term which arises due to the isotropic part of lensing potential. In Sect. 3, we estimate the modification to this term in presence of the inhomogeneous term. In Sect. 4, we obtain the expressions for the CMB power spectrum. In Sect. 5, we conclude.

\section{Gravitational lensing effect on two-point correlation functions in the presence of dipole anisotropy}

The observed CMB spectrum is different from that produced at LSS due to the lensing effect. These are related as [24]

$$
\begin{aligned}
X(\boldsymbol{\theta}) & =\tilde{X}(\boldsymbol{\theta}+\delta \boldsymbol{\theta}) \\
& =\frac{1}{(2 \pi)^{2}} \int d^{2} \boldsymbol{l} e^{i \boldsymbol{l} .(\boldsymbol{\theta}+\delta \boldsymbol{\theta})} \tilde{X}(\boldsymbol{l}),
\end{aligned}
$$

where, $X(\boldsymbol{\theta})$ is the observed physical quantity, such as the temperature $T(\boldsymbol{\theta})$, the Stokes parameters $Q(\boldsymbol{\theta})$ and $U(\boldsymbol{\theta})$ etc., and $\tilde{X}(\boldsymbol{\theta}+\delta \boldsymbol{\theta})$ is the corresponding physical quantity produced at LSS. Writing these variables in the explicit form, we have,

$$
\begin{aligned}
T(\boldsymbol{\theta}) & =\tilde{T}(\boldsymbol{\theta}+\delta \boldsymbol{\theta}) \\
& =\frac{1}{(2 \pi)^{2}} \int d^{2} \boldsymbol{l} e^{i \boldsymbol{l} \cdot(\boldsymbol{\theta}+\delta \boldsymbol{\theta})} \tilde{T}(\boldsymbol{l}), \\
Q(\boldsymbol{\theta}) & =\tilde{Q}(\boldsymbol{\theta}+\delta \boldsymbol{\theta}) \\
& =\frac{1}{(2 \pi)^{2}} \int d^{2} \boldsymbol{l} e^{i \boldsymbol{l} \cdot(\boldsymbol{\theta}+\delta \boldsymbol{\theta})} \tilde{Q}(\boldsymbol{l}),
\end{aligned}
$$

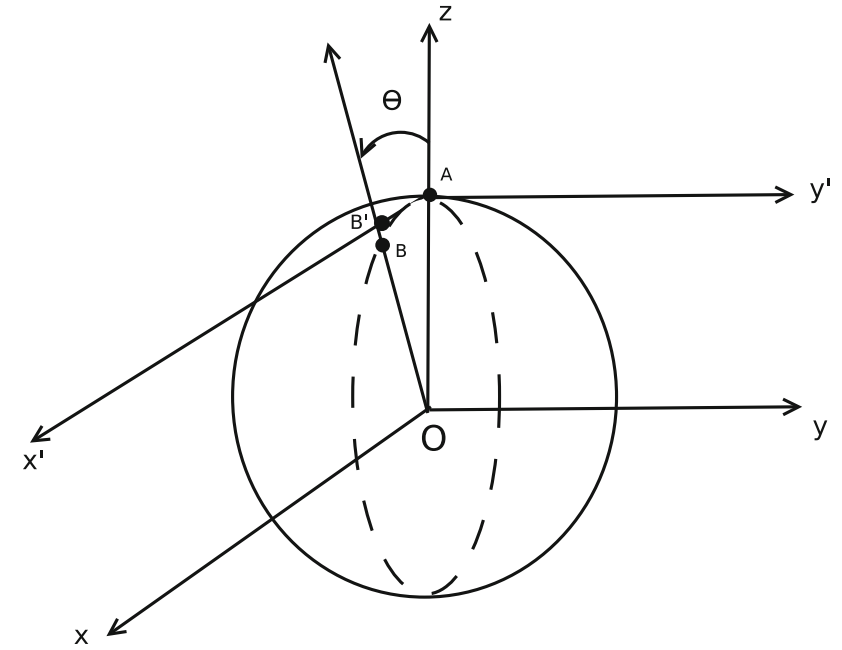

Fig. 1 In this plot the observer is located at $O$. We compute the correlations of CMB observables, such as temperature and Stokes parameters, between two points $A$ and $B$. The coordinate system is chosen such that point $A$ is at pole and $B$ lies in the $x-z$ plane. The projection of point $\mathrm{B}$ on a plane tangent at $A$ is $\mathrm{B}^{\prime}$. Projection of $\mathrm{A}$ itself is at $\mathrm{A}$. Both projected points lie on the $\mathrm{X}^{\prime}$ axis

$$
\begin{aligned}
= & \frac{1}{(2 \pi)^{2}} \int d^{2} \boldsymbol{l} e^{i l \cdot(\boldsymbol{\theta}+\delta \boldsymbol{\theta})} \\
& \times\left[\tilde{E}(\boldsymbol{l}) \cos \left(2 \phi_{\boldsymbol{l}}\right)-\tilde{B}(\boldsymbol{l}) \sin \left(2 \phi_{\boldsymbol{l}}\right)\right], \\
U(\boldsymbol{\theta})= & \tilde{U}(\boldsymbol{\theta}+\delta \boldsymbol{\theta}) \\
= & \frac{1}{(2 \pi)^{2}} \int d^{2} \boldsymbol{l} e^{i \boldsymbol{l} \cdot(\boldsymbol{\theta}+\delta \boldsymbol{\theta})} \tilde{U}(\boldsymbol{l}) \\
= & \frac{1}{(2 \pi)^{2}} \int d^{2} \boldsymbol{l} e^{i \boldsymbol{l} \cdot(\boldsymbol{\theta}+\delta \boldsymbol{\theta})} \\
& \times\left[\tilde{E}(\boldsymbol{l}) \sin \left(2 \phi_{\boldsymbol{l}}\right)+\tilde{B}(\boldsymbol{l}) \cos \left(2 \phi_{\boldsymbol{l}}\right)\right],
\end{aligned}
$$

where, the standard definitions of $Q(\boldsymbol{l})$ and $U(\boldsymbol{l})$ are used. Using Eqs. (3), (4) and (5), we can compute correlation function of such quantities at two points $\theta_{1}$ and $\theta_{2}$. In general, these two points can be anywhere on the two dimensional plane in small scale limit formalism. However for simplicity, we rotate our frame such that projection of these two points fall on $x^{\prime}$-axis (see Fig. 1). We can also shift $\theta_{1}$ at $\theta_{1}=0$ or $\theta_{2}$ at $\theta_{2}=0$ keeping other point at the separation of $\theta$. Now, the correlation function corresponding to the temperature is given by,

$$
\begin{aligned}
C_{T}(\theta) & =\left\langle T\left(\boldsymbol{\theta}_{1}\right) T\left(\boldsymbol{\theta}_{2}\right)\right\rangle \\
& =\left\langle\tilde{T}\left(\boldsymbol{\theta}_{1}+\delta \boldsymbol{\theta}_{1}\right) \tilde{T}\left(\boldsymbol{\theta}_{2}+\delta \boldsymbol{\theta}_{2}\right)\right\rangle \\
& =\int \frac{d^{2} \boldsymbol{l}}{(2 \pi)^{2}} e^{i l \theta \cos \left(\phi_{l}\right)}\left\langle e^{i \boldsymbol{l} \cdot\left(\delta \boldsymbol{\theta}_{1}-\delta \boldsymbol{\theta}_{2}\right)}\right\rangle C_{\tilde{T} l},
\end{aligned}
$$

where, we have used

$$
\left\langle\tilde{Y}(\boldsymbol{l}) \tilde{Y}\left(\boldsymbol{l}^{\prime}\right)\right\rangle=(2 \pi)^{2} \tilde{C}_{Y \boldsymbol{l}} \delta^{2}\left(\boldsymbol{l}-\boldsymbol{l}^{\prime}\right) .
$$

The vector quantity $\boldsymbol{l}$, which is conjugate to $\boldsymbol{\theta}$, makes the angle $\phi_{l}$ with the $x$-axis. In the similar way, we obtain the 
following relation for $C_{Q}(\theta)$ and $C_{U}(\theta)$,

$$
\begin{aligned}
C_{Q}(\theta)= & \int \frac{d^{2} \boldsymbol{l}}{(2 \pi)^{2}} e^{i l \theta \cos \phi_{l}}\left\langle e^{i \boldsymbol{l} \cdot\left(\delta \boldsymbol{\theta}_{1}-\delta \boldsymbol{\theta}_{2}\right)}\right\rangle \\
& \times\left[C_{\tilde{E} l} \cos ^{2}\left(2 \phi_{\boldsymbol{l}}\right)+C_{\tilde{B} l} \sin ^{2}\left(2 \phi_{\boldsymbol{l}}\right)\right] \\
C_{U}(\theta)= & \int \frac{d^{2} \boldsymbol{l}}{(2 \pi)^{2}} e^{i l \theta \cos \phi_{l}}\left\langle e^{i \boldsymbol{l} \cdot\left(\delta \boldsymbol{\theta}_{1}-\delta \boldsymbol{\theta}_{2}\right)}\right\rangle \\
& \times\left[C_{\tilde{E} l} \sin ^{2}\left(2 \phi_{l}\right)+C_{\tilde{B} l} \cos ^{2}\left(2 \phi_{l}\right)\right] .
\end{aligned}
$$

The quantity $\left\langle e^{i \boldsymbol{l} \cdot\left(\delta \boldsymbol{\theta}_{1}-\delta \boldsymbol{\theta}_{2}\right)}\right\rangle$ is important since we are interested in the weak lensing effect in the presence of the dipole anisotropy. We compute this quantity following Ref. [24] including the contribution from the superhorizon mode given in Eq. (1). Let us define the deflection angles as $\alpha=\delta \theta_{1}$ and $\alpha^{\prime}=\delta \theta_{2}$. The deflection angle is given by,

$\alpha=\nabla_{\hat{n}} \psi(\hat{n})$,

where, $\nabla_{\hat{n}}$ is angular derivative, $\hat{n}$ is direction of observation and $\psi(\hat{n})$ is lensing potential and it is connected to gravitational potential $\psi\left(\chi \hat{n}, \eta_{0}-\chi\right)$ via,

$\psi(\hat{\eta}) \equiv-2 \int_{0}^{\chi^{*}} d \chi \frac{f_{K}\left(\chi^{*}-\chi\right)}{f_{K}\left(\chi^{*}\right) f_{K}(\chi)} \psi\left(\chi \hat{n}, \eta_{0}-\chi\right)$.

Here $\chi^{*}$ is conformal distance of LSS from us. In our case, the gravitational potential has two parts, the homogeneous part $\boldsymbol{\psi}^{h}$ and the part $\boldsymbol{\psi}^{d}$ corresponding to the dipole distribution:

$\boldsymbol{\psi}=\boldsymbol{\psi}^{h}+\boldsymbol{\psi}^{d}$

Expanding $\boldsymbol{\psi}^{h}$ by Fourier expansion, we have

$\boldsymbol{\psi}(\theta)=\frac{1}{(2 \pi)^{2}} \int d^{2} \boldsymbol{l} e^{i \boldsymbol{l} \cdot \boldsymbol{\theta}} \boldsymbol{\psi}_{\boldsymbol{l}}^{h}+\boldsymbol{\psi}^{d}$.

Now we calculate the correlation tensor,

$\left\langle\alpha_{i} \alpha_{j}^{\prime}\right\rangle=\left\langle\nabla_{i} \psi \nabla_{j} \psi^{\prime}\right\rangle$

We obtain

$$
\begin{aligned}
\left\langle\alpha_{i} \alpha_{j}^{\prime}\right\rangle & =\left\langle\nabla_{i} \psi \nabla_{j} \psi^{\prime}\right\rangle, \\
& =\left\langle\nabla_{i} \psi^{h} \nabla_{j} \psi^{\prime h}\right\rangle+\left\langle\nabla_{i} \psi^{d} \nabla_{j} \psi^{\prime d}\right\rangle, \\
& =\int \frac{d^{2} \boldsymbol{l}}{(2 \pi)^{2}} \boldsymbol{l}_{i} \boldsymbol{l}_{j} e^{i \boldsymbol{l} \cdot \boldsymbol{\theta}} C_{l}^{\psi \psi^{\prime}}+\left\langle\nabla_{i} \psi^{d} \nabla_{j} \psi^{\prime d}\right\rangle,
\end{aligned}
$$

where, $\left\langle\psi_{\boldsymbol{l}}^{h} \psi_{\boldsymbol{l}^{\prime}}^{h *}\right\rangle=(2 \pi)^{2} \delta^{2}\left(\boldsymbol{l}-\boldsymbol{l}^{\prime}\right) C_{l}^{\psi \psi^{\prime}}$ is used. The potential $\psi^{d}\left(\eta^{*}, \hat{n}\right)$ is computed later and it is uncorrelated to $\psi^{h}$. In Eq. (14), we denote the first term, which is the homogeneous part, by $\left\langle\alpha_{i}^{h} \alpha_{j}^{\prime h}\right\rangle$. The correlation function $\left\langle\alpha_{i}^{h} \alpha_{j}^{\prime h}\right\rangle$ should be proportional to $\delta_{i j}$ and the trace-free tensor is given by $\left\langle\hat{r}_{i} \hat{r}_{j}\right\rangle=\hat{r}_{i} \hat{r}_{j}-\frac{1}{2} \delta_{i j}$. Using this fact, the first, homogeneous part, simplifies as

$$
\int \frac{d^{2} \boldsymbol{l}}{(2 \pi)^{2}} \boldsymbol{l}_{i} \boldsymbol{l}_{j} e^{i \boldsymbol{l} \cdot \boldsymbol{\theta}} C_{l}^{\psi \psi^{\prime}}=\frac{1}{2} C_{g l} \delta_{i j}-C_{g l, 2}\left\langle\hat{r}_{i} \hat{r}_{j}\right\rangle,
$$

where,

$$
\begin{aligned}
C_{g l}(\theta) & =\int \frac{d l}{2 \pi} l^{3} C_{l}^{\psi \psi^{\prime}} J_{0}(l \theta), \\
C_{g l, 2}(\theta) & =\int \frac{d l}{2 \pi} l^{3} C_{l}^{\psi \psi^{\prime}} J_{2}(l \theta) .
\end{aligned}
$$

We next consider the term $\left\langle e^{i l \cdot\left(\alpha-\alpha^{\prime}\right)}\right\rangle$ in which we are interested in order to understand the lensing effects including the dipole distribution. It can be shown that [1]

$\left\langle e^{i l \cdot\left(\alpha-\alpha^{\prime}\right)}\right\rangle=e^{-\frac{1}{2}\left\langle\left[l \cdot\left(\alpha-\alpha^{\prime}\right)\right]^{2}\right\rangle}$.

Expanding the term $\left\langle\left[l \cdot\left(\alpha-\alpha^{\prime}\right)\right]^{2}\right\rangle$, we have

$$
\begin{aligned}
\left\langle\left[l \cdot\left(\alpha-\alpha^{\prime}\right)\right]^{2}\right\rangle= & l^{i} l^{j}\left\langle\left(\alpha-\alpha^{\prime}\right)_{i}\left(\alpha-\alpha^{\prime}\right)_{j}\right\rangle \\
= & l^{i} l^{j}\left[\left\langle\alpha_{i} \alpha_{j}\right\rangle+\left\langle\alpha_{i}^{\prime} \alpha_{j}^{\prime}\right\rangle-2\left\langle\alpha_{i} \alpha_{j}^{\prime}\right\rangle\right] \\
= & l^{i} l^{j}\left[\left\langle\alpha_{i} \alpha_{j}\right\rangle+\left\langle\alpha_{i}^{\prime} \alpha_{j}^{\prime}\right\rangle-2\left\langle\alpha_{i} \alpha_{j}^{\prime}\right\rangle\right]^{h} \\
& +l^{i} l^{j}\left[\left\langle\alpha_{i} \alpha_{j}\right\rangle+\left\langle\alpha_{i}^{\prime} \alpha_{j}^{\prime}\right\rangle-2\left\langle\alpha_{i} \alpha_{j}^{\prime}\right\rangle\right]^{d} .
\end{aligned}
$$

We obtain the terms due to homogeneous part as well as dipole part, since $\left\langle\alpha_{i} \alpha_{j}\right\rangle,\left\langle\alpha_{i}^{\prime} \alpha_{j}^{\prime}\right\rangle$ and $\left\langle\alpha_{i} \alpha_{j}^{\prime}\right\rangle$ all have two parts as in Eq. (14). The homogeneous part becomes

$$
\begin{aligned}
& \left\langle\left[l \cdot\left(\alpha-\alpha^{\prime}\right)\right]^{2}\right\rangle^{h} \\
& \quad=l^{2}\left[C_{g l}(0)-C_{g l}(\theta)+\cos \left(2 \phi_{l}\right) C_{g l, 2}(\theta)\right], \\
& \quad=l^{2}\left[\sigma_{0}^{2}(\theta)+\sigma_{2}^{2}(\theta) \cos \left(2 \phi_{l}\right)\right],
\end{aligned}
$$

where, we define $\sigma_{0}^{2}(\theta)=C_{g l}(0)-C_{g l}(\theta)$ and $\sigma_{2}^{2}(\theta)=$ $C_{g l, 2}$. These notations, $\sigma_{0}^{2}(\theta)$ and $\sigma_{2}^{2}(\theta)$, have been used in the literature [24].

\section{Dipole anisotropy correction}

In this section, we estimate first the correction to $\langle[l \cdot(\alpha-$ $\left.\left.\left.\alpha^{\prime}\right)\right]^{2}\right\rangle$ due to the dipole distribution. In Eq. (14), the additional second term is due to the dipole. To calculate it, we expand $\psi^{d}$ in 3-dimensional Fourier space as follows [25],

$$
\begin{aligned}
\psi^{d}\left(\eta^{*}, \hat{n}\right)= & -2 \int_{0}^{\chi^{*}} d \chi_{1} \frac{f_{K}\left(\chi^{*}-\chi_{1}\right)}{f_{K}\left(\chi^{*}\right) f_{K}\left(\chi_{1}\right)} \\
& \times \int \frac{d^{3} k}{(2 \pi)^{3}} \boldsymbol{\psi}^{d}(\boldsymbol{k}) e^{i k \cdot\left(\eta_{0}-\eta_{1}\right) \hat{n}} T_{\psi}\left(\eta_{1}, k\right) .
\end{aligned}
$$

The dipole distribution $\boldsymbol{\psi}^{d}(\boldsymbol{k})$ is the Fourier transform of the potential given in Eq. (1) and is given by,

$\boldsymbol{\psi}^{d}(\boldsymbol{k})=\frac{\beta}{2 i}(2 \pi)^{3}\left[\delta^{3}\left(\boldsymbol{k}-\kappa_{s} \hat{x}\right)-\delta^{3}\left(\boldsymbol{k}+\kappa_{s} \hat{x}\right)\right]$,

where, $\beta$ and $\kappa_{s}$ are two parameters which define the dipole. As explained earlier the coordinate system has been chosen such that the dipole is aligned in $x$-direction. We keep $\psi^{d}$ in generalized form of 3D. Now, we redefine the momentum $k$ 
in terms of $l$, by $\vec{l}=\chi^{*} \vec{k}, \int d^{3} k \boldsymbol{\psi}^{d}(k) \rightarrow \int d^{3} l \boldsymbol{\psi}^{d}(l)$ and $\frac{\eta_{0}-\eta}{\chi^{*}}=\frac{\chi}{\chi^{*}}=\omega$. In this definition,

$$
\begin{aligned}
\frac{\boldsymbol{\psi}^{d}(k)}{\chi^{* 3}} & =\frac{\beta}{2 i} \frac{(2 \pi)^{3}}{\chi^{* 3}}\left[\delta^{3}\left(\boldsymbol{k}-\kappa_{s} \hat{x}\right)-\delta^{3}\left(\boldsymbol{k}+\kappa_{s} \hat{x}\right)\right] \\
& =\frac{\beta}{2 i}(2 \pi)^{3}\left[\delta^{3}\left(\chi^{*} \boldsymbol{k}-\chi^{*} \kappa_{s} \hat{x}\right)-\delta^{3}\left(\chi^{*} \boldsymbol{k}+\chi^{*} \kappa_{s} \hat{x}\right)\right], \\
& =\frac{\beta}{2 i}(2 \pi)^{3}\left[\delta^{3}(\boldsymbol{l}-\kappa \hat{x})-\delta^{3}(\boldsymbol{l}+\kappa \hat{x})\right]=\psi^{d}(l),
\end{aligned}
$$

where, $\kappa=\chi^{*} \kappa_{s}$. Under this definition, Eq. (21) can be written as,

$$
\begin{aligned}
\psi^{d}\left(\eta^{*}, \hat{n}\right)= & -2 \int_{0}^{\chi^{*}} d \chi_{1} \frac{f_{K}\left(\chi^{*}-\chi_{1}\right)}{f_{K}\left(\chi^{*}\right) f_{K}\left(\chi_{1}\right)} \\
& \times \int \frac{d^{3} l}{(2 \pi)^{3}} \psi^{d}(l) e^{i l . \hat{n} \omega} T_{\psi}\left(\eta_{1}, l\right) .
\end{aligned}
$$

We assume that the transfer function is nearly unity at large scale and set it as unity in the further calculation. We can now simplify the term $\left\langle\nabla_{i} \psi^{d} \nabla_{j} \psi^{\prime d}\right\rangle$ as follows,

$$
\begin{aligned}
& \left\langle\nabla_{i} \psi^{d}\left(\eta^{*}, \hat{n_{1}}\right) \nabla_{j} \psi^{* d}\left(\eta^{*}, \hat{n_{2}}\right)\right\rangle \\
& =4 \int_{0}^{\chi^{*}} \int_{0}^{\chi^{*}} d \chi_{1} d \chi_{2} \frac{\left(\chi^{*}-\chi_{1}\right)}{\left(\chi^{*} \chi_{1}\right)} \frac{\left(\chi^{*}-\chi_{2}\right)}{\left(\chi^{*} \chi_{2}\right)} \\
& \quad \times \iint d^{3} l d^{3} l^{\prime} \omega_{1} \omega_{2} l_{i} l_{j}^{\prime}\left(\frac{\beta^{2}}{4}\right) \\
& \quad \times\left[\delta^{3}(\vec{l}-\kappa \hat{x}) \delta^{3}\left(\overrightarrow{l^{\prime}}-\kappa \hat{x}\right)-\delta^{3}(\vec{l}-\kappa \hat{x}) \delta^{3}\left(\overrightarrow{l^{\prime}}+\kappa \hat{x}\right)\right. \\
& \left.\quad-\delta^{3}(\vec{l}+\kappa \hat{x}) \delta^{3}\left(\overrightarrow{l^{\prime}}-\kappa \hat{x}\right)+\delta^{3}(\vec{l}+\kappa \hat{x}) \delta^{3}\left(\overrightarrow{l^{\prime}}+\kappa \hat{x}\right)\right] \\
& \quad \times e^{i \hat{l} . \hat{n}_{1} \omega_{1}} e^{-i \hat{l}^{\prime} \cdot \hat{n}_{2} \omega_{2}} .
\end{aligned}
$$

Here, $\eta$ is the conformal time at the point of observation. The operator $\nabla_{i}$ has two components corresponding to the directions $x$ and $y$ and in the flat sky approximation these are equivalent to $\frac{\partial}{\partial n_{x}}$ and $\frac{\partial}{\partial n_{y}}$ respectively. Here $n_{x}$ and $n_{y}$ are $\mathrm{x}$ and y components of $\hat{n}$, the unit vector in the direction of observation from us. The two points of observation could be anywhere on the sphere, however, we consider these points on the great circle in the $x-z$ plane. The unit direction $\hat{n}$, which is given by

$\hat{n}=\sin \theta \cos \phi \hat{x}+\sin \theta \sin \phi \hat{y}+\cos \theta \hat{z}$

becomes $\sin \theta \hat{x}+\cos \theta \hat{z}(\phi=0)$ in this case. The dipole correlation tensor turns out to be (see Appendix A)

$$
\begin{aligned}
& \left\langle\nabla_{i} \psi^{d}\left(\eta^{*}, \hat{n_{1}}\right) \nabla_{j} \psi^{* d}\left(\eta^{*}, \hat{n_{2}}\right)\right\rangle \\
& \quad=2 \beta^{2} \delta_{i}^{x} \delta_{j}^{x}\left[\frac{1-\cos [\kappa \sin \theta]}{\sin ^{2} \theta}\right] .
\end{aligned}
$$

In the notation of $\alpha_{i}$,

$$
\left\langle\alpha_{i}^{d} \alpha_{j}^{\prime d}\right\rangle=2 \beta^{2} \delta_{i}^{x} \delta_{j}^{x}\left[\frac{1-\cos [\kappa \sin \theta]}{\sin ^{2} \theta}\right],
$$

and so,

$$
\left\langle\alpha_{i}^{d} \alpha_{j}^{d}\right\rangle=\left\langle\alpha_{i}^{\prime d} \alpha_{j}^{\prime d}\right\rangle=\lim _{\theta \rightarrow 0}\left\langle\alpha_{i}^{d} \alpha_{j}^{\prime d}\right\rangle=\beta^{2} \kappa^{2} \delta_{i}^{x} \delta_{j}^{x} .
$$

Our motivation is to calculate $\left\langle\left|\vec{l} .\left(\vec{\alpha}_{i}-\vec{\alpha}_{i}^{\prime}\right)\right|^{2}\right\rangle^{d}$. Keeping all quantities in hand, in flat-sky approximation ( $l$ lying in $x-y$ plane), we can simplify this quantity as,

$$
\begin{aligned}
& \left\langle\left|\vec{l} .\left(\vec{\alpha}-\vec{\alpha}^{\prime}\right)\right|^{2}\right\rangle^{d} \\
& =l^{i} l^{j}\left[\left\langle\alpha_{i}^{d} \alpha_{j}^{d}\right\rangle+\left\langle\alpha_{i}^{\prime d} \alpha_{j}^{\prime d}\right\rangle-2\left\langle\alpha_{i}^{d} \alpha_{j}^{\prime d}\right\rangle\right] \\
& =\beta^{2} l^{2}\left(1+\cos 2 \phi_{l}\right)\left[\kappa^{2}-2 \frac{(1-\cos [\kappa \sin \theta])}{\sin ^{2} \theta}\right] .
\end{aligned}
$$

\section{CMB power spectrum}

We observe that the dipole anisotropy modifies the quantities $C_{g l}(0)-C_{g l}(\theta)\left(\equiv \sigma_{0}^{2}\right)$ and $C_{g l, 2}\left(\equiv \sigma_{2}^{2}\right)$ with equal weight. Therefore, $\left\langle e^{i l \cdot\left(\alpha-\alpha^{\prime}\right)}\right\rangle$ turns out to be,

$\left\langle e^{i l \cdot\left(\alpha-\alpha^{\prime}\right)}\right\rangle=\exp \left\{-\frac{l^{2}}{2}\left[\sigma_{0 T}^{2}+\cos \left(2 \phi_{l}\right) \sigma_{2 T}^{2}\right]\right\}$,

where, we define new quantities $\sigma_{0 T}^{2}$ and $\sigma_{2 T}^{2}$ as,

$$
\begin{aligned}
\sigma_{0 T}^{2} & =C_{g l}(0)-C_{g l}(\theta)+\beta^{2}\left[\kappa^{2}-2 \frac{(1-\cos [\kappa \sin \theta])}{\sin ^{2} \theta}\right] \\
& =\sigma_{0}^{2}+\sigma_{d}^{2},
\end{aligned}
$$

and,

$$
\begin{aligned}
\sigma_{2 T}^{2} & =C_{g l, 2}(\theta)+\beta^{2}\left[\kappa^{2}-2 \frac{(1-\cos [\kappa \sin \theta])}{\sin ^{2} \theta}\right] \\
& =\sigma_{2}^{2}+\sigma_{d}^{2} .
\end{aligned}
$$

Here, $\sigma_{d}^{2}$ is given by

$\sigma_{d}^{2}=\beta^{2}\left[\kappa^{2}-2 \frac{(1-\cos [\kappa \sin \theta])}{\sin ^{2} \theta}\right]$.

In Eq. (31), we note that the term is similar to what we get in the standard lensing. The only difference here is that all the effects of anisotropy are absorbed in $\sigma_{0 T}^{2}$ and $\sigma_{2 T}^{2}$. This facilitates us in using further construction of standard weak lensing. Therefore, from Eqs. (6) and (8), we obtain,

$$
\begin{aligned}
C_{T}(\theta)= & \int \frac{l d l}{2 \pi} C_{\tilde{T} l}\left[J_{0}(l \theta)\left[1-\frac{l^{2}}{2} \sigma_{0 T}^{2}(\theta)\right]\right. \\
& \left.+\frac{l^{2}}{2} \sigma_{2 T}^{2}(\theta) J_{2}(l \theta)\right], \\
C_{Q}(\theta)+C_{U}(\theta)= & \int \frac{l d l}{2 \pi}\left(C_{\tilde{E} l}+C_{\tilde{B} l}\right) \\
& \times\left[J_{0}(l \theta)\left[1-\frac{l^{2}}{2} \sigma_{0 T}^{2}(\theta)\right]\right.
\end{aligned}
$$



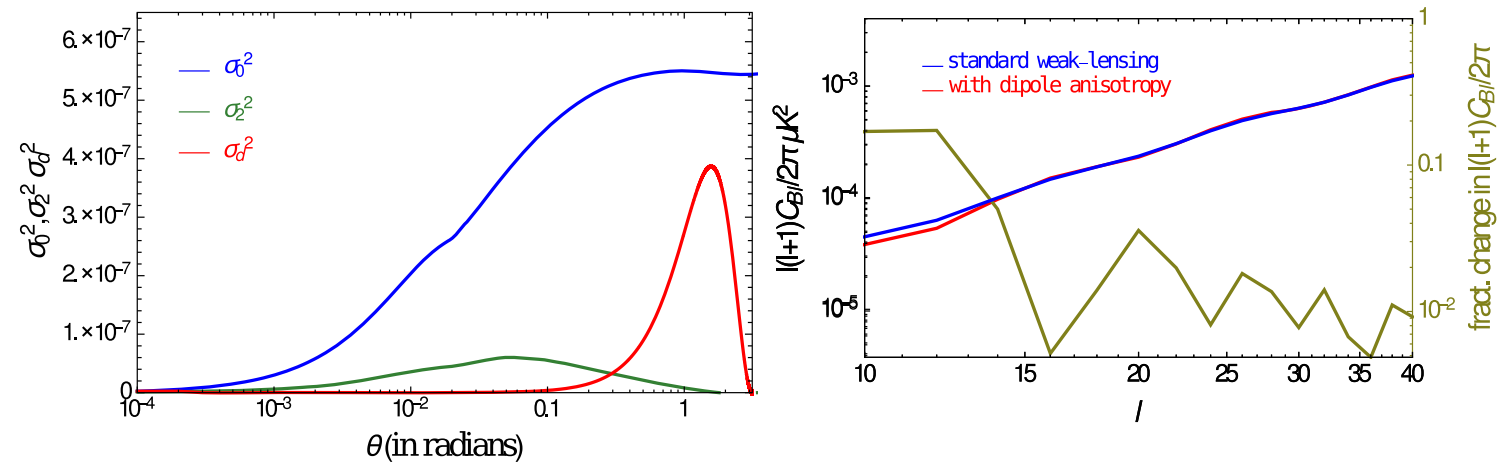

Fig. 2 The left panel shows plot for $\sigma_{0}^{2}, \sigma_{2}^{2}$ and $\sigma_{d}^{2}$. On the right panel, blue curve is B-mode power spectrum for the standard weak lensing and red curve is for the weak lensing with the dipole distribution. The fractional change in $\frac{l(l+1) C_{B}}{2 \pi}$ due to dipole is also plotted on right side vertical axis
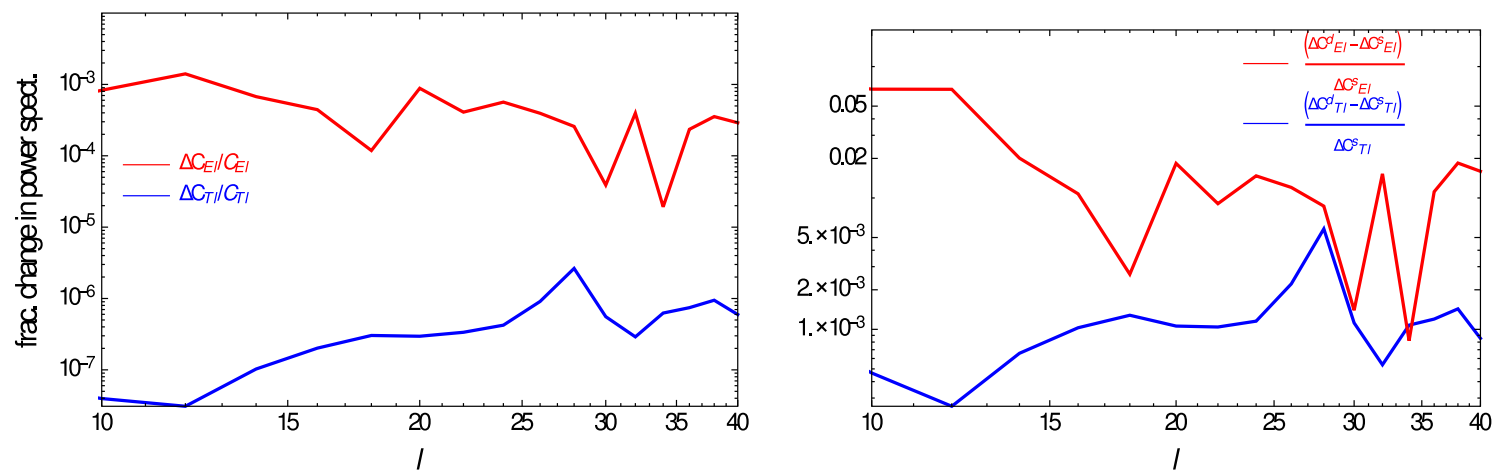

Fig. 3 Left: fractional contribution to E- and T-mode due to lensing including the contribution from the long wavelength mode (1). Right: the fractional change in power spectrum due to the long wavelength mode in comparison to standard lensing

$$
\begin{aligned}
& \left.+\frac{l^{2}}{2} \sigma_{2 T}^{2}(\theta) J_{2}(l \theta)\right], \\
C_{Q}(\theta)-C_{U}(\theta)= & \int \frac{l d l}{2 \pi}\left(C_{\tilde{E} l}-C_{\tilde{B} l}\right) \\
& \times\left[J_{4}(l \theta)\left[1-\frac{l^{2}}{2} \sigma_{0 T}^{2}(\theta)\right]\right. \\
& \left.+\frac{l^{2}}{4} \sigma_{2 T}^{2}(\theta)\left[J_{2}(l \theta)+J_{6}(l \theta)\right]\right] .
\end{aligned}
$$

We can now estimate the power spectrum in Fourier space from widely used definitions,

$$
\begin{aligned}
C_{T l}= & 2 \pi \int_{0}^{\pi} \theta d \theta C_{T}(\theta) J_{0}(l \theta), \\
C_{E l}= & 2 \pi \int_{0}^{\pi} \theta d \theta\left[\left[C_{Q}(\theta)+C_{U}(\theta)\right] J_{0}(l \theta)\right. \\
& \left.+\left[C_{Q}(\theta)-C_{U}(\theta)\right] J_{4}(l \theta)\right], \\
C_{B l}= & 2 \pi \int_{0}^{\pi} \theta d \theta\left[\left[C_{Q}(\theta)+C_{U}(\theta)\right] J_{0}(l \theta)\right. \\
& \left.-\left[C_{Q}(\theta)-C_{U}(\theta)\right] J_{4}(l \theta)\right] .
\end{aligned}
$$

From Eqs. (35) and (36), we can write the expressions for all the modified power spectra which reveal how these differ from the primordial power. These are as follows,

$$
\begin{aligned}
& C_{T l}=C_{\tilde{T} l}+\mathcal{W}_{1 l}^{l^{\prime}} C_{\tilde{T} l^{\prime}}, \\
& C_{E l}=C_{\tilde{E} l}+\frac{1}{2}\left[\mathcal{W}_{1 l}^{l^{\prime}}+\mathcal{W}_{2 l}^{l^{\prime}}\right] C_{\tilde{E} l^{\prime}}+\frac{1}{2}\left[\mathcal{W}_{1 l}^{l^{\prime}}-\mathcal{W}_{2 l}^{l^{\prime}}\right] C_{\tilde{B} l^{\prime}}, \\
& C_{B l}=C_{\tilde{B} l}+\frac{1}{2}\left[\mathcal{W}_{1 l}^{l^{\prime}}-\mathcal{W}_{2 l}^{l^{\prime}}\right] C_{\tilde{E} l^{\prime}}+\frac{1}{2}\left[\mathcal{W}_{1 l}^{l^{\prime}}+\mathcal{W}_{2 l}^{l^{\prime}}\right] C_{\tilde{B} l^{\prime}},
\end{aligned}
$$

where we sum implicitly over all $l^{\prime}$ and $\mathcal{W}_{1 l}^{l^{\prime}}$ and $\mathcal{W}_{2 l}^{l^{\prime}}$ are defined as,

$$
\begin{aligned}
\mathcal{W}_{1 l}^{l^{\prime}}= & \frac{l^{\prime 3}}{2} \int_{0}^{\pi} \theta d \theta J_{0}(l \theta)\left[\sigma_{2 T}^{2}(\theta) J_{2}\left(l^{\prime} \theta\right)-\sigma_{0 T}^{2}(\theta) J_{0}\left(l^{\prime} \theta\right)\right], \\
\mathcal{W}_{2 l}^{l^{\prime}}= & \frac{l^{\prime 3}}{2} \int_{0}^{\pi} \theta d \theta J_{4}(l \theta) \\
& \times\left[\frac{1}{2} \sigma_{2 T}^{2}(\theta)\left[J_{2}\left(l^{\prime} \theta\right)+J_{6}\left(l^{\prime} \theta\right)\right]-\sigma_{0 T}^{2}(\theta) J_{4}\left(l^{\prime} \theta\right)\right] .
\end{aligned}
$$

We note that in Eq. (37), the modified power spectra are determined by functions $W_{1 l}^{l^{\prime}}, W_{2 l}^{l^{\prime}}$ and $W_{3 l}^{l^{\prime}}$ which depend on $\sigma_{0 T}^{2}$ and $\sigma_{2 T}^{2}$. Since theoretically, the primordial $C_{\tilde{B} l}$ has nearly zero contribution, the correction to $C_{\tilde{E} l}$ is proportional to $W_{1 l}^{l^{\prime}}+W_{2 l}^{l^{\prime}}$ and the only contribution to $C_{B l}$ comes from the correction which is proportional to $W_{1 l}^{l^{\prime}}-W_{2 l}^{l^{\prime}}$. In 

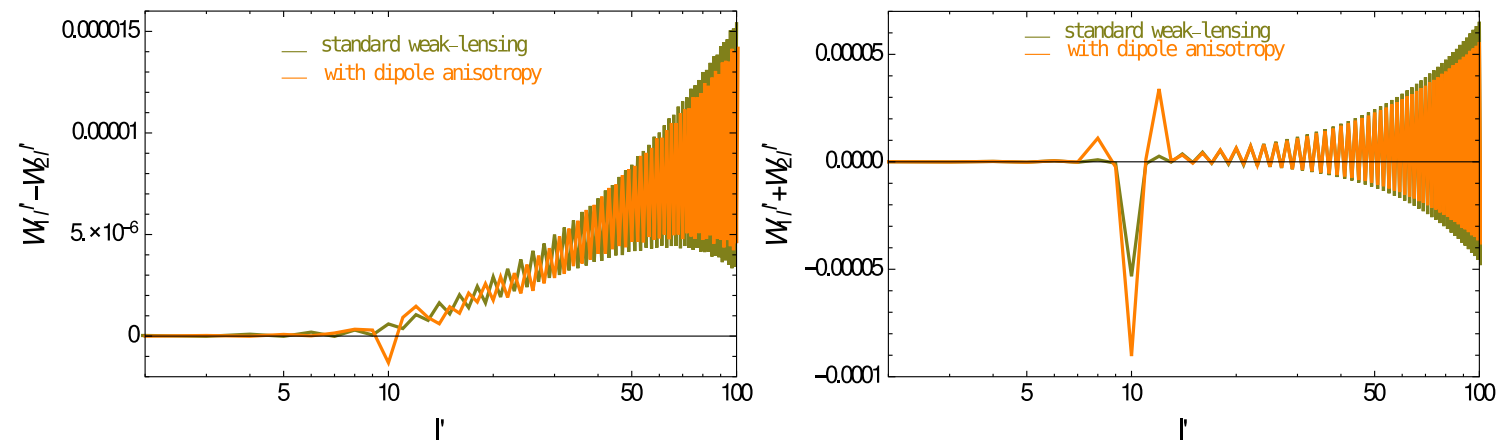

Fig. 4 The $l^{\prime}$ dependence of $W_{1 l}^{l^{\prime}}-W_{2 l}^{l^{\prime}}$ and $W_{1 l}^{l^{\prime}}+W_{2 l}^{l^{\prime}}$ for $l=10$ is shown in the left and right panels respectively

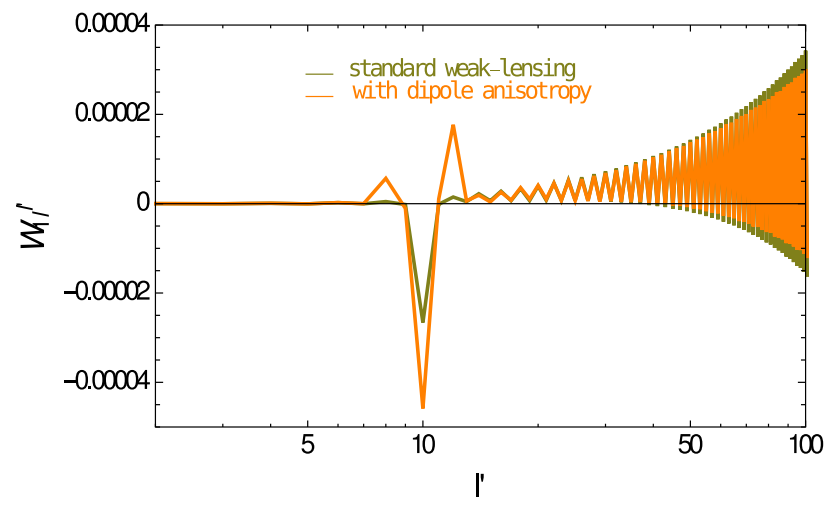

Fig. 5 The plot of $W_{1 l}^{l^{\prime}}$ with respect to $l^{\prime}$ for $l=10$

Fig. 2, on the left panel, we plot $\sigma_{0}^{2}, \sigma_{2}^{2}$ which are due to standard weak lensing. The function $\sigma_{d}^{2}$ due to dipole distribution is also plotted. We emphasize here that the term $\sigma_{d}$ is due to superhorizon mode in gravitational potential perturbation. As mentioned in the introduction, such a term can explain the observed dipole anisotropy in CMB power spectrum. One of the mechanism to produce such a large scale mode is multi-field inflation. For example, the curvaton model can explain the observed deviation in power spectrum [3]. The deviation of background value of scalar field across the observable Universe plays important role in generating power asymmetry. Fitting the observed values leads to the constraint $\left|\kappa_{s}^{3} \beta\right| \lesssim 1.26 \times 10^{-5} H_{0}^{3}$, where, $H_{0}$ is the Hubble constant $[3,18]$. We considered $\beta=1$ and $\kappa=0.046$ which satisfies $\left|\kappa_{s}^{3} \beta\right| \leq 1.26 \times 10^{-5} H_{0}^{3}$ or $\left|\kappa^{3} \beta\right| \leq 10^{-4}$ [18]. This provides an estimate of maximum possible change that can arise due to the dipole. We observe a reasonable value of $\sigma_{d}^{2}$ comparable to $\sigma_{0}^{2}$. In Fig. 3, on left panel, we plot the fractional changes $\left(\frac{\Delta C_{E l}}{C_{E l}}, \frac{\Delta C_{T l}}{C_{T l}}\right)$ due to weak lensing with dipole distribution. These are very small due to presence of the leading order terms $\tilde{C}_{E l}$ and $\tilde{C}_{T l}$. The term $W_{1 l}^{l^{\prime}}+W_{2 l}^{l^{\prime}}$ is oscillatory as well as roughly symmetric (see right panel of Fig. 4). Therefore, we do not get much contribution to the $C_{E l}$ due to the dipole distribu- tion. This can be seen on right panel of Fig. 3 where we plot $\left(\Delta C_{E l}^{d}-\Delta C_{E l}^{s}\right) / \Delta C_{E l}^{s}$ and $\left(\Delta C_{T l}^{d}-\Delta C_{T l}^{s}\right) / \Delta C_{T l}^{s}$, where $\Delta C_{E l}^{d}, \Delta C_{T l}^{d}$ are changes due to weak lensing in the presence of dipole and $\Delta C_{E l}^{s}, \Delta C_{T l}^{s}$ are changes due to standard lensing. Similarly, $W_{1 l}^{l^{\prime}}$ forms a roughly symmetric pattern (see Fig. 5) and it does not give much change to $C_{T l}$ in the standard weak lensing modification due to dipole anisotropy. The fractional changes observed in lensing for $C_{E l}$ and $C_{T l}$ are shown in the left panel of Fig. 3. Those values at $l=10$ are around $10^{-3}$ and $10^{-7}$ respectively. Fractional contributions from dipole are shown in right panel of the plot. Those values are approximately 0.05 and $10^{-3}$ respectively. So, in total, dipole gives very tiny change in these spectra, e.g. for $C_{E l}$ it is $\sim 10^{-5}$.

The contribution of $\sigma_{d}^{2}$ appears in the B-mode spectrum. The dipole distribution marginally decreases its value due to the presence of function $W_{1 l}^{l^{\prime}}-W_{2 l}^{l^{\prime}}$. In the right panel of Fig. 2, we plot the $B$-mode power spectrum including the dipole contribution. We also show its absolute fractional change on right side vertical axis in this plot. The standard weak lensing result is shown for comparison. In plotting the lensed $C_{B l}$, we use unlensed $\tilde{C}_{B l}=0$ and $\tilde{C}_{E l}$ from CAMB package. We restrict this curve for $l>10$ for which the Flat sky approximation is expected to be reliable [26]. We find that the dipole contribution leads to a small downward shift of the spectrum at low values of $l$. The shift is large at low $l$ and roughly $1 \%$ at $l$ of order 40 . Hence it is small but not negligible. At higher values $l \geq 40$, the fraction change becomes smaller and becomes $\sim 0.1 \%$ at $l \sim 100$. In Fig. 4 , on the left panel, we note that $W_{1 l}^{l^{\prime}}-W_{2 l}^{l^{\prime}}$ is oscillatory and mainly lie in positive-value quadrant. Due to this asymmetry, dipole distribution leads to a modification of $C_{B l}$.

\section{Conclusions}

We generalized the formalism of weak lensing by adding a superhorizon mode in the gravitational potential. Such a 
mode leads to a dipole modulation in the CMB power spectrum as well as a dipole anisotropy in the large scale structures. We estimated the effect of lensing on the CMB power spectrum in this model. We followed the approach of the flat sky approximation. Weak lensing mixes E and B mode and hence we necessarily find some contribution to $C_{B l}$ due to weak lensing. The correction to $C_{B l}$ is proportional to $\tilde{C}_{E l}$. We observed that adding dipole anisotropy changes $C_{B l}$ for the lower range of $l$. However, dipole anisotropy does not lead to an appreciable change in $C_{E l}$, since the correction is much smaller than the leading order term $\tilde{C}_{E l}$. We did not even observe much change for lower $l$ in the standard weak lensing modification due to the dipole anisotropy, since $W_{1 l}^{l^{\prime}}+W_{2 l}^{l^{\prime}}$ has an approximately symmetric pattern. The same argument applies to $C_{T l}$. In the case of $C_{B l}$ we obtain a correction since in this case the leading order $\tilde{C}_{B l}$ is zero and $W_{1 l}^{l^{\prime}}-W_{2 l}^{l^{\prime}}$ is not symmetric. For this case we computed the maximum possible correction to $C_{B l}$ by fixing the direction of dipole along the line on which, the projections of the observation points lie. A more reliable calculation would use the spherical harmonics approach. Flat sky approximation and the spherical harmonics approach deviate below $l=10$ [26]. Thus, our calculation is reliable for $l>10$, where we still find an observable correction to $C_{B l}$. Due to dipole term, the low multipoles get a significant correction for the B-mode. We may detect the resulting lensing effect as the polarization noise in CMB observations planned for near future is expected to be around one-fifth of lensing-B mode spectrum [27]. Our calculation would be useful in extracting the information about B-mode from the future experiments as long as dipole asymmetry persists in observations.

Acknowledgements The work of Prabhakar Tiwari is supported by NSFC Grants 11720101004 and 11673025, and the National Key Basic Research and Development Program of China (No. 2018YFA0404503). The work of Pankaj Jain is supported by a grant from the Science and Engineering Research Board (SERB), Government of India.

Data Availability Statement This manuscript has no associated data or the data will not be deposited [Authors' comment: The manuscript has no associated data.]

Open Access This article is distributed under the terms of the Creative Commons Attribution 4.0 International License (http://creativecomm ons.org/licenses/by/4.0/), which permits unrestricted use, distribution, and reproduction in any medium, provided you give appropriate credit to the original author(s) and the source, provide a link to the Creative Commons license, and indicate if changes were made.

Funded by SCOAP ${ }^{3}$.

\section{Appendix A: Dipole correlation tensor}

The momentum integrals in Eq. (25) contains four terms, we calculate these one by one. The first term is given by,

$$
\begin{aligned}
& \frac{\beta^{2}}{4} \iint d^{3} l d^{3} l^{\prime} \omega_{1} \omega_{2} l_{i} l_{j}^{\prime}\left[\delta^{3}(\vec{l}-\kappa \hat{x}) \delta^{3}\left(\overrightarrow{l^{\prime}}-\kappa \hat{x}\right)\right] e^{i \hat{l} \cdot \hat{n}_{1} \omega_{1}} e^{-i \hat{l^{\prime}} \cdot \hat{n}_{2} \omega_{2}} \\
& \quad=\frac{\beta^{2}}{4} \omega_{1} \omega_{2} \kappa^{2} \delta_{i}^{x} \delta_{j}^{x} e^{i \kappa\left(\omega_{1} \sin \theta_{1}-\omega_{2} \sin \theta_{2}\right)},
\end{aligned}
$$

similarly second, third and last terms can be written as,

$$
\begin{aligned}
- & \frac{\beta^{2}}{4} \iint d^{3} l d^{3} l^{\prime} \omega_{1} \omega_{2} l_{i} l_{j}^{\prime}\left[\delta^{3}(\vec{l}-\kappa \hat{x}) \delta^{3}\left(\overrightarrow{l^{\prime}}+\kappa \hat{x}\right)\right] e^{i \hat{l} \cdot \hat{n_{1}} \omega_{1}} e^{-i \hat{l^{\prime}} \cdot \hat{n_{2}} \omega_{2}} \\
= & \frac{\beta^{2}}{4} \omega_{1} \omega_{2} \kappa^{2} \delta_{i}^{x} \delta_{j}^{x} e^{i \kappa\left(\omega_{1} \sin \theta_{1}+\omega_{2} \sin \theta_{2}\right)}, \\
- & \frac{\beta^{2}}{4} \iint d^{3} l d^{3} l^{\prime} \omega_{1} \omega_{2} l_{i} l_{j}^{\prime}\left[\delta^{3}(\vec{l}+\kappa \hat{x}) \delta^{3}\left(\overrightarrow{l^{\prime}}-\kappa \hat{x}\right)\right] e^{i \hat{l} \cdot \hat{n_{1}} \omega_{1}} e^{-i \hat{l^{\prime}} \cdot \hat{n_{2}} \omega_{2}} \\
= & \frac{\beta^{2}}{4} \omega_{1} \omega_{2} \kappa^{2} \delta_{i}^{x} \delta_{j}^{x} e^{-i \kappa\left(\omega_{1} \sin \theta_{1}+\omega_{2} \sin \theta_{2}\right)},
\end{aligned}
$$

and

$$
\begin{aligned}
& \frac{\beta^{2}}{4} \iint d^{3} l d^{3} l^{\prime} \omega_{1} \omega_{2} l_{i} l_{j}^{\prime}\left[\delta^{3}(\vec{l}+\kappa \hat{x}) \delta^{3}\left(\overrightarrow{l^{\prime}}+\kappa \hat{x}\right)\right] e^{i \hat{l} . \hat{n_{1}} \omega_{1}} e^{-i \hat{l^{\prime}} \cdot \hat{n_{2}} \omega_{2}} \\
& \quad=\frac{\beta^{2}}{4} \omega_{1} \omega_{2} \kappa^{2} \delta_{i}^{x} \delta_{j}^{x} e^{-i \kappa\left(\omega_{1} \sin \theta_{1}-\omega_{2} \sin \theta_{2}\right)},
\end{aligned}
$$

respectively. Summing all the terms, we obtain the total contribution, which is given by,

$\beta^{2} \omega_{1} \omega_{2} \kappa^{2} \delta_{i}^{x} \delta_{j}^{x} \cos \left(\kappa \omega_{1} \sin \theta_{1}\right) \cos \left(\kappa \omega_{2} \sin \theta_{2}\right)$

As already mentioned, we may shift one point to the pole. Here we accomplish this shift by making $\theta_{2} \rightarrow 0$. We can also now redefine $\theta_{1}$ as only $\theta$, therefore, above term can be written as,

$\beta^{2} \omega_{1} \omega_{2} \kappa^{2} \delta_{i}^{x} \delta_{j}^{x} \cos \left(\kappa \omega_{1} \sin \theta\right)$.

Now we integrate this term with respect to conformal time to simplify Eq. (25) by recalling $\omega=\frac{\chi}{\chi^{*}}$. This leads to,

$$
\begin{aligned}
& \left\langle\nabla_{i} \psi^{d}\left(\eta^{*}, \hat{n_{1}}\right) \nabla_{j} \psi^{* d}\left(\eta^{*}, \hat{n_{2}}\right)\right\rangle \\
& =2 \beta^{2} \delta_{i}^{x} \delta_{j}^{x}\left[\frac{1-\cos [\kappa \sin \theta]}{\sin ^{2} \theta}\right] .
\end{aligned}
$$

\section{References}

1. A. Lewis, A. Challinor, Phys. Rept. 429, 1 (2006). https://doi.org/ 10.1016/j.physrep.2006.03.002. arXiv:astro-ph/0601594

2. C. Gordon, W. Hu, D. Huterer, T.M. Crawford, Phys. Rev. D 72, 103002 (2005). https://doi.org/10.1103/PhysRevD.72.103002. arXiv:astro-ph/0509301

3. A.L. Erickcek, M. Kamionkowski, S.M. Carroll, Phys. Rev. D 78, 123520 (2008). https://doi.org/10.1103/PhysRevD.78.123520. arXiv:0806.0377 [astro-ph]

4. C.L. Bennett, et al. [WMAP Collaboration], Astrophys. J. Suppl. 208, 20 (2013). https://doi.org/10.1088/0067-0049/208/2/ 20. arXiv:1212.5225 [astro-ph.CO]

5. M. Tegmark, A. de Oliveira-Costa, A. Hamilton, Phys. Rev. D 68, 123523 (2003). https://doi.org/10.1103/PhysRevD.68.123523. arXiv:astro-ph/0302496

6. C.J. Copi, D. Huterer, G.D. Starkman, Phys. Rev. D 70, 043515 (2004). https://doi.org/10.1103/PhysRevD.70.043515. arXiv:astro-ph/0310511 
7. K. Land, J. Magueijo, Phys. Rev. Lett. 95, 071301 (2005). https://doi.org/10.1103/PhysRevLett.95.071301. arXiv:astro-ph/0502237

8. P. Vielva, E. Martinez-Gonzalez, R.B. Barreiro, J.L. Sanz, L. Cayon, Astrophys. J. 609, 22 (2004). https://doi.org/10.1086/ 421007. arXiv:astro-ph/0310273

9. P. Mukherjee, Y. Wang, Astrophys. J. 613, 51 (2004). https://doi. org/10.1086/423021. arXiv:astro-ph/0402602

10. M. Cruz, E. Martinez-Gonzalez, P. Vielva, L. Cayon, Mon. Not. R. Astron. Soc. 356, 29 (2005). https://doi.org/10.1111/j.1365-2966. 2004.08419.x. arXiv:astro-ph/0405341

11. H.K. Eriksen, F.K. Hansen, A.J. Banday, K.M. Gorski, P.B. Lilje, Astrophys. J. 605, 14 (2004). Erratum: [Astrophys. J. 609, 1198 (2004)]. https://doi.org/10.1086/382267. arXiv:astro-ph/0307507

12. P.A.R. Ade, et al. [Planck Collaboration], Astron. Astrophys. A 571, 23 (2014). https://doi.org/10.1051/0004-6361/201321534. arXiv:1303.5083 [astro-ph.CO]

13. P. Jain, J.P. Ralston, Mod. Phys. Lett. A 14, 417 (1999). https://doi. org/10.1142/S0217732399000481. arXiv:astro-ph/9803164

14. J.P. Ralston, P. Jain, Int. J. Mod. Phys. D 13, 1857 (2004). https:// doi.org/10.1142/S0218271804005948. arXiv:astro-ph/0311430

15. D. Hutsemékers, A\&A 332, 410-428 (1998)

16. P. Jain, G. Narain, S. Sarala, Mon. Not. R. Astron. Soc. 347, 394 (2004). https://doi.org/10.1111/j.1365-2966.2004.07169.x. arXiv:astro-ph/0301530
17. A.R. Liddle, M. Cortês, Phys. Rev. Lett. 111(11), 111302 (2013). https://doi.org/10.1103/PhysRevLett.111.111302. arXiv:1306.5698 [astro-ph.CO]

18. S. Ghosh, Phys. Rev. D 89, 063518 (2014). https://doi.org/10.1103/ PhysRevD.89.063518. arXiv:1309.6547 [astro-ph.CO]

19. R. Kothari, S. Ghosh, P.K. Rath, G. Kashyap, P. Jain, Mon. Not. R. Astron. Soc. 460(2), 1577 (2016). https://doi.org/10.1093/mnras/ stw1039. arXiv:1503.08997 [astro-ph.CO]

20. S. Ghosh, R. Kothari, P. Jain, P.K. Rath, JCAP 1601(01), 046 (2016). https://doi.org/10.1088/1475-7516/2016/01/046. arXiv:1507.04078 [astro-ph.CO]

21. S. Ghosh, P. Jain, arXiv:1807.02359 [astro-ph.CO]

22. A. Blanchard, J. Schneider, Astron. Astrophys. 184, 1 (1987)

23. U. Seljak, Astrophys. J. 463, 1 (1996). https://doi.org/10.1086/ 177218. arXiv:astro-ph/9505109

24. M. Zaldarriaga, U. Seljak, Phys. Rev. D 58, 023003 (1998). https:// doi.org/10.1103/PhysRevD.58.023003. arXiv:astro-ph/9803150

25. B. Yu, T. Lu, Astrophys. J. 698, 1771 (2009). https://doi.org/10. 1088/0004-637X/698/2/1771. arXiv:0903.4519 [astro-ph.CO]

26. W. Hu, Phys. Rev. D 62, 043007 (2000). https://doi.org/10.1103/ PhysRevD.62.043007. arXiv:astro-ph/0001303

27. A. Challinor, et al. [CORE Collaboration], JCAP 1804(04), 018 (2018). https://doi.org/10.1088/1475-7516/2018/04/018. arXiv:1707.02259 [astro-ph.CO] 